\title{
Tiếp Cận Hệ Thống Trong Điều Khiền Các Quá Trình Công Nghệ
}

\author{
Nguyên Văn Châu \\ Viện "Tin Họ \\ Viện Khoa Học Việt Nam
}

\section{Mờ đầu}

Cùng với sự bùng nổ về thông tin và công nghệ ngày nay thuật ngữ công nghệ đã thâm nhập vào nhiều mặt của đời sống xã hội. Nó gắn liền với hoạt động của con người vào những mục đích nhất định. Việc nghiên cưu bản chấtcủa quá trĩnh công nghệ do đó là vấn để triết học còn phát triển. Tuy nhiên tồn tại một lớp khá rộng cắc quá trình công nghệ có đặc thù là nhằm mục đích thực hiện việc làm ra sàn phẩm cho tiêu dùng xã hội dưới dạng vật chất và năng lượng. Nói cách khác mục đích hoạt động của con người ở đây được thể hiện trong sản phẩm cuối dưới dạng vật chất và năng lượng.

Trong bài này tác giầ chỉ giới hạn xét đến khả năng điểu khiển lợp quá trỉnh công nghệ nói trên. Đề phân biệt khái niệm quá trình công nghệ ở đây được kỉ hiệu QTCN. Các định nghĩa trong bài này chỉ dùng trong phạm vi giới hạn đó.

\section{Một vài khái niệm}

1 - Hiện tượng kỹ thuật - là hiện tượng lí hoá sinh do con người thực hiện theo các thiết kế chủ quan đối với các đối tượng lao động và có sử dụng vào mục đích đó các công cụ và phương tiện kỹ thuật.

2 - QTCN - là một tập hợp các hiện tượng kỹ thuật có liên hệ với nhau, là kh âu sơ cấp tạó ra các giá trị vật chát đảm bảo thực hiện việc làm ra các sản phẩm cần thiết cho tờn tại xã hội. Trong QTCN các hiện tượng kỹ thuật liên hệ với nhau trực tiếp và xác định qua các sản phâm cuối cụ thể (cần hiểu ở đây khái niệm sản phẩm cuối là sản phăm dưới dạng vật chất và năng lượng đã có thể đi vào tiêu dùng hoặc cũng có thể là nguyên, nhiên liệu, năng lượng cho các QTCN khác). QTCN do đó là quá trình kŷ thuật có chứa đựng các quan hệ kinh tế - xã hội.

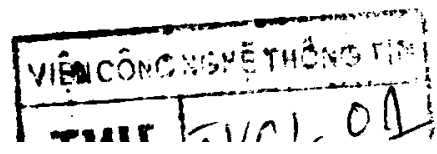


Với ý nghia đó, cùng với mức độ phát triển khoa học kŷ thuật tương ứng, khái, niệm kinh diền về quá trình công nghệ thường chì các quá trình kỹ thuật không phức tạp lắm được thực hiện với một vài đối tượng công nghệ không nhiều lắm và chủ yếu là được thực hiện trên một thiết bị công nghệ chính và có thể cùng vơi một số không lớn lắm các thiết bị phụ. Các QTCN có thể chỉ là một quá trỉnh kỹ thuật hoặc một số không lớn lắm các quá trình kỹ thuật có liên hệ với nhau. Trong trường hợp chung có thể diễn đạt trạng thái cuà QTCN dưới dạng số lượng cuả một vài chỉ tiêu độc lập $X$ nào đó đói với sản phâm (vi dụ: áp suất, nhiệt độ, điện áp, tần số, nồng độ, độ $\mathrm{PH}, \ldots$ ) trong đó mỡi chì tiêu được biễu diễn bằng véc tơ $n$ chiều:

$$
X(t)=\left\{x_{i}(t)\right\}_{i \in N}
$$

$x_{i}(t)$ - biến trạng thái; không gian trạng thái là không gian chứa véc tơ $X(t)$ và nói chung là không gian xác suấl $(\mathcal{X}, S, \mu)$ trong đó $S$ là $\sigma$ - đại số cuả các tập con $x ; \mu$ - độ đo xác suất.

,Dạng chuần cuả phương trình trạng thái khi đó là

$$
\dot{X}(t)=f(X(t), U(t), t, \xi) .
$$

Phương trình quan sát hệ

$$
Y(t)=g(X(t), U(t), \eta)
$$

$U(t)$ - véc tơ đièu khiển, là véc tơ các thông số quản lí được (hoặc dẫn xuất cuả chúng); $\xi$, $\eta$ - các véc tơ nhiễu nói chung có thứ nguyên $x \leq n$.

\section{Tiếp cận hệ thống trong điều khiển các QTCN}

Nhu câu xã hội ngày càng tăng về số lượng và cả về chất lượng. Điểu đó dẫn đến sự xuất hiện nhiểu công nghệ mới và các khả năng thực hiện chúng ( thiết bị công nghẹe, vật liệu, công nghệ, thiét bị và phương pháp xử lý thông tin điêu khiên). Ví dụ: trong quá trình lưu hóa một sản phẩm cao su có thẻ nhận sản phẩm với chát lượng như nhau bằng nhiều phương án (công nghệ): 10 phút ở 150 đọ $\mathrm{C}, 8$ phút ơ 180 độ $\mathrm{C}$ hoặc 6 phút ơ 210 dộ C. Ví dụ khác: năng xuất cuà một quá trinh công nghệ hóa chát:

$$
V=k \cdot C_{L}^{l} C_{M}^{m} \cdots
$$

$C_{L}, C_{M}$ - nồng đọ mol cuà các hoá chất tham gia phàn ứng; $l, m, \ldots$ - hệ số phụ thuộc dạng phản ứng

$$
k=f(\theta)
$$

$\theta$ - nhiệt độ phản ứng.

Rõ ràng là có nhiểu khả năng (công nghệ) đề thực hiện quá trình đó.

Ví dụ khác: để cung cấp một lượng năng lượng nhiệt, điện cho lưới năng lượng với chất lượng và số lượng nhất định $(U, \omega, P$ - điện áp, tần số, công suất) có thể có nhiều phương án khác nhau thay đổi các thông số trên sơ đồ nhiệt cuả nhà máy nhiệt điện ( thay đổi phân phối phụ tải giữa các khối lò máy, thay đổi các thông số hổi nhiệt, gia nhiệt, thay đổi cung cấp nhiên liệu, ... ). 
Việc xuất hiện nhiều công nghệ mới và khả năng thực hiện kỹ thuật đông thời cũng dẫn đến phức tạp hoá bản thân các đối tượng công nghệ cũng như các mối quan hệ giữa các thiết bị công nghệ chính và phụ. Thứ nguyên các mô hỉnh toán học các QTCN do đó tăng lên rất lớn. Diều đó trên thực tể gây nhiểu khó khăn cho việc nghiên cứu cũng như thực hiện kỹ thuật các hệ điều khièn chúng.

Tiệm cận hệ thống cho phép xem các quá trỉnh kỹ thuật là các quá trình cơ sở (QTCS). Các QTCS có thẻ đóng vai trò chính hoặc phụ trong QTCN. Các ví dụ dó (chính hoặc phụ) có thể thay đổi trong quá trỉnh tiến hành QTCN tương ứng với sự thay đổi trọng số cuả chúng trong giới hạn loại

$$
\sum_{k \in N} \gamma_{k}=1
$$

Trong QTCN, các QTCS liên hệ trực tiếp với nhau (liên hệ nối tiếp một chiểu hoặc hai chiều) hoặc liên hệ với nihau qua sản phẩm cuối (liên hệ song song hoặc hỗn hợp). Các QTCS đảm bảo chủ yếu mặt chất lượng nên có tính kỹ thuật. Các mối liên hệ chủ yếu trong QTCN được xác định thông qua các thông số công nghệ và các chỉ tiêu đối với sản phẩm. Đối với toàn bộ QTCN bởi vì nó luôn gắn liền với khái niệm sản phẩm cuối nên nó thực hiện cả hai mặt cuả sản phẩm: số lượng (phi kỹ thuật - năng suất, các loại chi phí,...) và chất lượng (kỹ thuật - độ chính xác công ñghệ). Các quan hệ chủ yếu trong QTCNं được xác định thông qua các thông số công nghệ và các chỉ tiêu cuả sản phẩm cuối bao gồm hai mặt: chất lượng và số lượng cuả sản phẩm cuối. Như vậy sản phẩm cuối là điều kiện tồn tại cuả QTCN và là sự thống nhất cuả hai mặt chất lượng và số lượng (vốn đối lập nhau) cuà QTCN.

Sự thay đôi trạng thái cuả QTCN được xác định bới sự thay đổi trạng thái cuá QTCS và sự thay đởi cuà các điều kiện thực hiện các QTCS. Như vậy nếu với khái niệm kinh điển QTCN được xem như một hệ kỹ thuật thi với quan điểm hệ thóng ngoài những đặc trưng kỹ thuật, QTCN còn mang những đặc trưng phi kỹ thuật (thường là những đặc trưng kinh tế). Do đó trong công nghiệp thông thường có thể xem các QTCN là các hệ kinh tế kỹthuật.

Từ đó, điều khiển QTCN là lựa chọn các thông số kiêm soát được sao cho có thể đưa QTCN đến trạng thái tối ưu theo ý nghĩa cuả cả hai mặt: chẩt lượng và số lượng cuả sản phẩm cuối.

Từ những phân tỉch cơ băn đó đi đến tiên đề:

Tiên đề - Không tồn tại QTCN mà không có sản phẩm cuối.

- QTCN là điêu khiên được.

và có các định nghĩa sau:

Dịnh nghia 1 QTCN là hệ kinh tế- kỹ thuật điều khiển được và được xác định bởi:

- mô hình cấu trúc:

Gỏm bộ bốn:

$$
\left(S,\left\{S_{k} \mid k=1,2, \ldots, M, N\right\}, \gamma, \beta\right)
$$

trong đó: $S$ đặc trưng cuả QTCN; $S_{k}$ - dặctrưng cuá QTCS ${ }_{k} ; k=1,2, \ldots, N ; \gamma=\left\{\gamma_{1}, \gamma_{2}, \ldots, \gamma_{N}\right\}$ - tập các liên hệ, trong đó $\gamma_{k}$ là tập các liên hệ cuả $\mathrm{QTCS}_{k}$ với các $\mathrm{QTCS}_{l \neq k}$ trong hệ; $\beta=$ 
$\left\{\beta_{1}, \beta_{2}, \ldots, \beta_{M}\right\}$ - tạ̣p các lièn hệ, trong đó $\beta_{k}$ là lièn hẹ trực tiếp giữa QTCS $_{k}$ với QTCN. Ngoài ra

$$
\beta \neq 0, \quad \text { nghĩa là } \exists \beta_{l}=\emptyset
$$

- Mô hỉnh chuyển pha:

Hệ các hàm chuyển trạng thải:

$$
\dot{X}_{k}(t)=F\left(\mathrm{Y}_{k}(t), U_{k}(t), \beta_{k}, \gamma_{k}, \xi_{k}\right) ; k=1,2, \ldots, N
$$

trong đó: $X_{k}$ - véc tơ trạng thái cuả QTCS $X_{k}: X_{k} \in X ; U_{k}$ - véc tơ điều khiển cuả QTCS $_{k}$ : $U_{k} \in U ; \xi_{k}$ - véc tơ nhiếu. Ngoài ra

$$
X \neq \emptyset, U \neq \emptyset, \gamma_{k}=g^{*}\left(X_{k}(t), \beta_{k}\right) ; \beta_{k}=g^{* *}\left(X_{k}(t), \gamma_{k}\right)
$$

Để minh họa hãy khảo sát một vài cấu trúc điển hỉnh san (hỉnh 1 và 3 ):

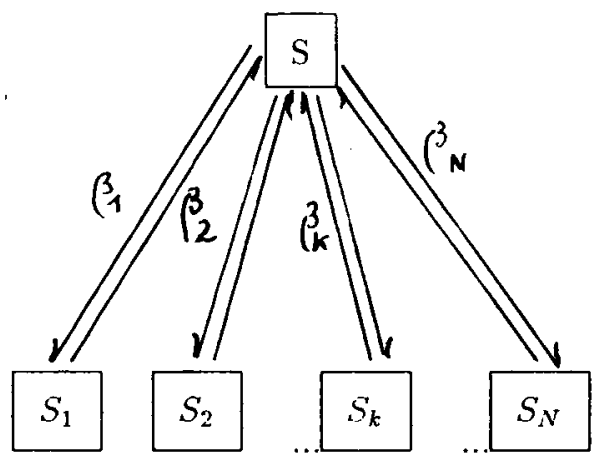

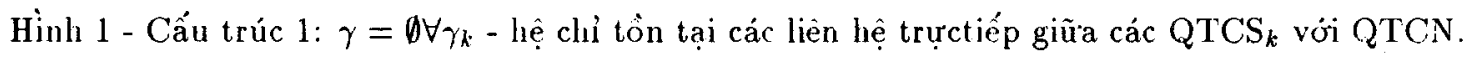

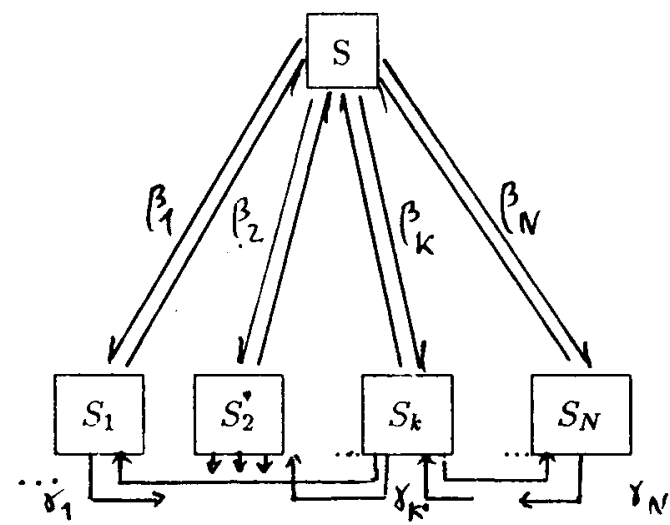

Hình 2 - Cấu trúc 2: $\gamma_{k} \neq \emptyset$ and $\beta_{k} \neq 0$ - giữa các QTCS tồn tại các liên hệ. 


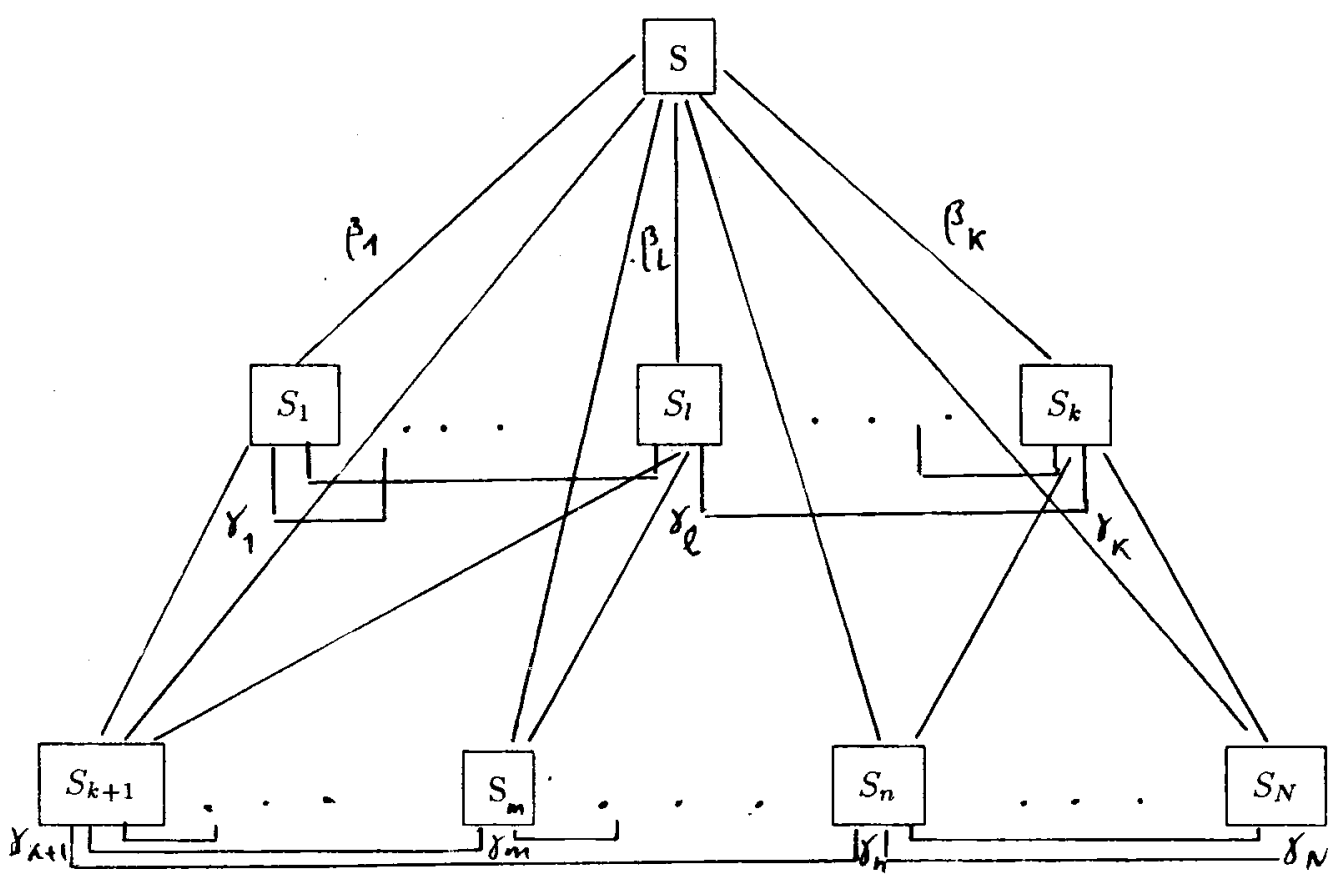

Hỉnh 3: - Cáu trúc 3: $\beta=\left\{\beta_{1}, \beta_{2}, \ldots, \beta_{N}\right\} ; \gamma=\left\{\gamma_{1}, \gamma_{2}, \ldots \gamma_{N}\right\}$ trong đó $\{\gamma, m, n=1,2, \ldots N\}$ - tập các chỉ số được ánh xạ tìr $\{1,2, \ldots, N\} \rightarrow\{1,2, \ldots, N\} ; \beta_{1}, \ldots, \beta_{k}, \ldots, \beta_{N} ; \exists \gamma_{k}: \gamma_{k} \neq \emptyset$

Cấu trúc này biêu diễn trường hợp có những QTCS chỉ liên hệ được với QTCN qua các QTCN khác do đó còn gọi là cấu trúc phân cấp. Khihi đó $\beta_{l}=\emptyset, S_{l}$ không có liên hệ trực tiếp với $S$ mà chỉ liên hệ phân cấp. Thông qua $s_{m \neq l}^{\prime \prime}$ xuát hiện phân cấp mói. Bởi vì $\gamma$ và $\beta$ là biện đởi động (7) trong quá trỉnh tién hành QTCN, do đó tỉnh phân cấp trong QTCN là đông.

Trong công nghệ việc đảm bảo chất lượng sản phảm thường được thực hiện bằng cách giữ ổn định một vài thông số công nghẹ gắn liền với các QTCS:

Gọi $V_{k}=V_{k}\left(X_{k}(t), U_{k}(t), t, \xi_{k}(t)\right)$ viết gọn là $V_{k}=V_{k}(t)$ là hàm biêu diễn các thông số công nghệ được điều khiển trong $\mathrm{QTCS}_{k}$ ta có:

Dịnh nghĩa 2 QTCS là hệ kỹ thuật $S_{k}$ được xác định từ định nghĩa 1 và

$$
S_{k}=f\left\{V_{k}\left(X_{k}, U_{k}, t, \xi_{k}\right): \exists k \in K, l \in K=\left\{1,2, \ldots, K^{*}\right\} \Rightarrow \lim _{t \rightarrow \infty}\left|V_{k}^{*}(t)-V_{k}(t)\right| \leq \epsilon\right.
$$

đ̛ đây $V_{k}(t)$ - thông số công nghệ được điều khiền; $V_{k}^{*}(t)$ - đánh giá cuả $V_{k}(t) ; \epsilon$ - ngưỡng cho trước tìy ý và xác định theo (III-3).

Dẽ dàng thấy rằng QTCN có cấu trúc 1 nếu thỏa mãn ĐN 1 và ĐN 2.

Định nghia 3 QTCS xác định bỏi ĐN 1 và ĐN 2 là có điều khiên và điều khiên được nếu trong đó

$$
\exists U_{k}: U_{k} \neq \emptyset \text {. }
$$

Dịnh nghĩa 4 QTCS là được điểu khiên tự trị nếu nó thoả mãn

1 - các điểu kiện (8), (9)

$2-\gamma_{k}=\emptyset$ 
Trong đó các điều kiện (8) và (9) khẳng định tính điểu khiển được cuả QTCS. Điều kiện (10) xác định tỉnh không có liên hệ trực tiếp với các $\mathrm{QTCS}_{l \neq k}$ cuà $\mathrm{QTCS}_{k}$.

- Trong hệ QTCN có $\beta \neq \emptyset$ và $\gamma \neq \emptyset \forall \gamma_{k}$, nghĩa là tất cả các QTCS đều được điều khiền tự trị thỉ ta gọi đó là QTCN được điều khiển tự trị. Một hệ như thế thoả mãn cấu trúc 1.

Từ các định nghĩa trên dẫn đến:

Định lý 1 ( Định lý tồn tại) Nếu $V$ là hàm đặc trưng các thông số cuả QTCN cẩn giữ ổn định hoặc giữ thay đởi theo một hàm cho trước thì t nhất $\exists S_{k}: S_{k}=f(V)$. Ơ đây hàm $f$ được xác dịh theo DN 2.

Chứng minh Nếu ngược lại, theo ĐN 2 ta có $S_{k} \neq \emptyset \forall k \Rightarrow X=\emptyset$ trái với (7) cuả DN 1.

Định lý 2 ( Định lý phân tích) Nếu $M>N$ thỉ QTCN có cấu trúc phân cấp nhiêu cấp: $n$

Chứng minh Nếu $n=1$ và $M>N$ thì ít nhất $\exists S_{k}$ mà trong đó $\beta_{k}^{*} \neq \beta_{k}^{* *}$ trái với DN 1.

Hệ quà 1 Néu $V_{k}$ là thông số cuả QTCN cần giữ ổ định hoặc giữ thay đồi theo một hàm cho trước thi ít uhất $\exists S_{k}: S_{k}=f\left(V_{k}\right)$ và $V_{k}=f\left(X_{k}, \beta_{k}\right)$.

Chứng minh Tì̀ định lý 1 dễ dàng thấy rằng khi đó $V_{k}=V$.

\section{Phân tích và tổng hợp}

Việc phân tích và tông hợp QTCN cơ bản là dựa vào mô hỉnh cấu trúc và mô hỉnh chuyển phía (ĐN 1 ).

Khi nghiên cứu cấu trúc QTCN buộc phải xem xét các tính chất macro cuả hệ trên cơ sở nghiên cưu các trạng thái macro và micro cuả chúng. Bởi vì tồn tại sự khác biệt vể các tính chất và nội dung kỹ thuật - kinh tế ngay trong bản thân đối tượng (QTCN) nên có thể xác định các trạng thái macro và micro trong những không gian khác nhau. Việc nghiên cứu các liên hệ giữa các trạng thái macro và micro là vấn đề cuả các đới tượng nghiên cứu (QTCN) cụ thế.

1 - Trang thái micro là trạng thái cuả hệ được xác định bởi các tọa độ và tốc độ (hoặc gia tốc) các biển. Việc xừ dụng các phương pháp phân tich micro (các phương pháp tần số, phương pháp phương trình trạng thái ... ) đổi với các QTCN ít phức tạp có thứ nguyên nhó hoặc đối với các QTCS thường rất có hiệu quả. Ở trạng thải micro, tư cách (trạng thái) cuả hệ được diễn đạt dười dạng tường minin quan hẹ cuà các thông số cấu trúc, các liên hệvà các tác đọng ngoài. Có thể xem trạng thái micro là hàm cuả các bién trạng thải và tốc độ (hoặc gia tốc) cuả chúng. Dạng hàm đó phụ thuộc cấu trúc hệ và các tác động ngoài.

Khi dó ta có

$$
X_{l}=G_{l}\left(x_{l}, u_{l}, \beta_{l}, \gamma_{l}, \xi, t\right)
$$

ơ đây $x_{l}=\left\{x_{l}^{1}, x_{l}^{2}, \ldots, x_{l}^{M}\right\}$ - véc tơ ngẫu nhiên các trạng thái micro với phân bố $p\left(x_{l}^{i}\right)$ và kỳ̀ vọng

$$
\bar{X}_{l}=\int_{M} x_{l}^{i} d p\left(x_{l}^{i}\right)
$$


$G_{1}$ - một vài hàm phân bố tính chất micro trong trạng thái macro cuả hệ. Việc xác định (11) phụ thuộc vào phương pháp phân tích.

Trường hợp việc nghiên cứu tỉnh chất micro cuả hệ (QTCN) tương úng với việc nghiên cứu tính chất cuả một QTCS nào đó theo một nghĩa nào đó (ví dụ khi xét một chì tiêu chất lượng cuả sản phẩm cuối mà chỉ tiên đó lại do tính chất cuả một QTCS nào đó quyết định) thì (11) có thể xác định từ (6) theo ĐN 1.

2- Trạng thái macro Nói chung, có thể mô hình hóa các QTCN (vỉ dụ các QTCN trong sản xuất năng lượng nhiệt điện, trong các nghành công nghiệp giấy, hóa dầu, ...) bằng các phương pháp phân tích micro. Tuy nhiên trên thực tế mô hỉnh hóa các QTCN phức tạp, các phương pháp đó tỏ ra ít hiệu quà. Việc áp dụng các phương pháp phân tích macro (ví dụ phương pháp entropi) [37], phương pháp tương quan, phương pháp tập mớ, ...) có thể chỉ ra các tính chất macro cần thiết mà không nhất thiết phải tính đến mọi tính chất micro cuả hệ. Tổ hợp nhiều trạng thái micro có thể dẫn đến một trạng thái macro. Nhưng điều đó không có nghĩa là trạng thái macro cuả QTCN bằng tông (hoặc tích) các trạng thái micro. Điều đó trên cơ sở loại bỏ các liên hệ yếu có thể cho phép đơn giản hóa đến một mức độ đáng kể cấu trúc hệ khi tổ hợp hệ điều khiển các QTCN. Như vậy trạng thái $X$ cuả QTCN là một vài kết quả mở rộng cuả các trạng thái micro cuả nó. Ngoài ra nó còn chứa các tính chất macró khác không mô hỉnh hóa được khi tiến hành nghiên cứu micro hoặc không tồn tại trong các trạng thái micro (người ta gọi nó là emergence tính trội $E$ cuá các hê thổng lớn và p̣ưuc tạp). Rõ ràng là: nếu

$$
\exists S: \forall \beta_{k}=\emptyset \Rightarrow n=\emptyset \& E=\emptyset \text {. }
$$

Từ (13) cho ta tính trội $E$ chỉ có thể được phát hiện và tôn tại trong macro trạng thái QTCN trong điêu khiện giữa chủ thể điêu khiển và đối tượng điêu khiển (micro trạng thái và các mức phân cấp macro thấp hơn) có trao đổi thông tin.

Từ cấu trúc 3 (cấu trúc tông quát) có thể rút ra sơ đồ phân cấp cuả trạng thái macro và được giới thiệu trên hỉnh 4 , ờ đây $n \geq 0$.

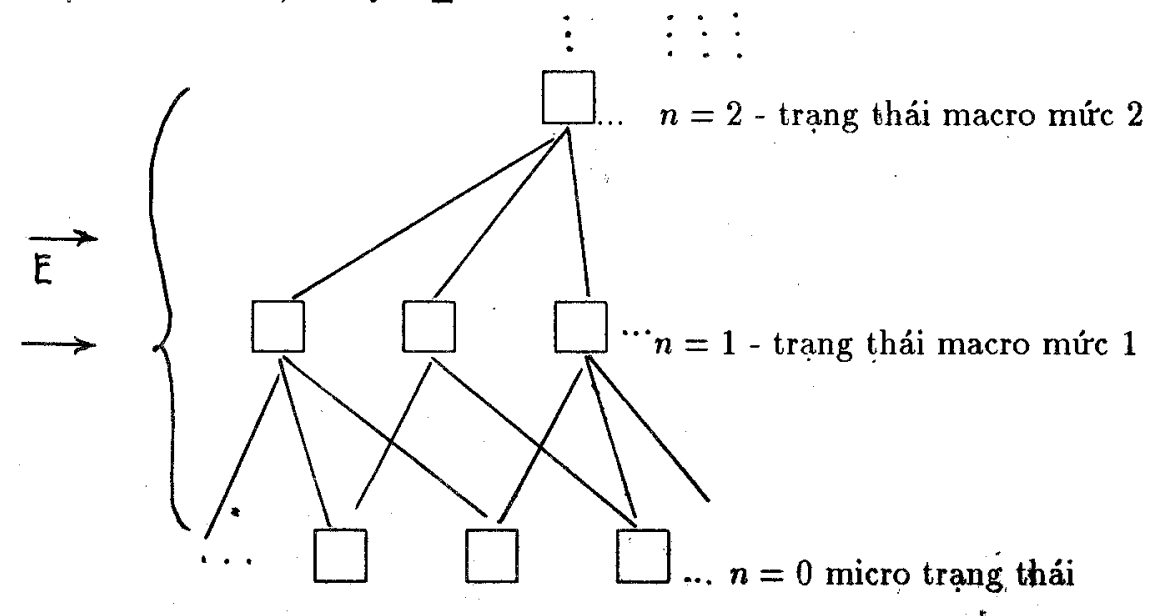

Hình 4. Tính phân cấp cuả trạng thái macro QTCN 
3- $\epsilon$ - ngưỡng. Điều khiển các QTCN cần được tiến hành theo những quan điểm chủ quan (hàm mục tiêu, các chỉ tiêu chất lượng, số lượng) cùng với các yéu tố tác động đến các quan điêm chủ quan đó ( truyền thống, thói quen, kinh nghiệm, sự đào tạo, mệnh lệnh từ trên, đường lối quy định trước, giá cả thị trường ...) trên cơ sở xem xẻt các yếu tổ khách quan hiện hữu. Do đó vai trò quan sát viên trong điều khiển các QTCN rất quan trọng. Quan sát viên có thể là người, là otomat, là máy tỉnh trong thành phân cơ cấu xử lí thông tin điều khiên và kiểm tra. Cạ́c yếu tố chủ quan đó có thể được diễn đạt dưới dạng số lượing qua khái niệm ngưỡng phân biệt cuả quan sát viên gọi là $\epsilon$ - ngưỡng [21,31].

Trong hệ được khảo sát, đối tượng quan sát được đặctrưng bởi một vài tập các biến (véc tơ $X_{I}$ ). Khi đó có thể lập mỗi véc tơ $X_{l}$ trong một trạng thái tương ứng với đối tượng được điều khiển sao cho tập tất cả các trạng thái có khả năng cuả đối tượng là dằng cấu với tập cuả mồi véc tơ $X_{l}$. Khái niệm đó là khải niệm chuẩn trong việc diễn đạt các hệ động học $[6,7]$. Có nghĩa là, khái niệm trạng thái (macro và micro) chỉ có thè có ý nghía với mỗi hệ đối tượng - quan sát viên cụ thể. Trong điều khiên QTCN nhờ $\epsilon$ - ngưỡng mà phân biệt được các trạng thái cuà hệ diễn đạt trong dạng cảc đặc trưng vể chất lượng và số lượng cuả sản phẩm cuối.

Vi dụ: Giả sử trạng thái cuả QTCN được xác định bởi các $\mathrm{DN}$ 1,2 và 3 . Một trong những nhiệm vụ điều khiên QTCN là ôn định thóng số công nghệ $V$. Khi đó chất lượng điều khiển đói với $V$ dược biểu diễn dưới dạng làm cực tiểu phiếm hàm $J_{k}$

$$
\min J_{k}=\min \Phi\left(V_{K}^{*}-V_{k}\right) .
$$

Diều đó cũng tương ưng với việc làm cực tiểu khoảng cách giữa hai điểm $V_{k}^{*}$ và $V_{k}$, nghia là

$$
\min J_{k}=\min \rho\left(V_{k}^{*}, V_{k}\right)=\min \left\{\rho\left(V_{k 1}^{*}, V_{k 1}\right), \rho\left(V_{k 2}^{*}, V_{k 2}, \ldots,\right\}\right. \text {. }
$$

Cho rằng QTCN được tiến hành một cảch hoàn chình nếu trị số bằng số cuả $J_{k}$ không vượt quá đại lượng cho phép một trị số $\epsilon_{k}>0$ nào đó,khi đó

$$
\rho\left(V_{k}^{*}, V_{k}\right)=\left|V_{k}^{*}-V_{k}\right| \leq \epsilon ; \epsilon_{k} \in \epsilon .
$$

Diêu đó có nghĩa là $\epsilon$ được chọn sao cho trạng thái macro cuả hệ không thay đổi khi hệ chưa ra khơi giởi hạn (16). (Ỏ đây $\epsilon$ - ngưỡng là tập các ngương quan sát theo khái niệm trên). Có nghĩa là $\epsilon_{k}$ là moduyn bé nhất cuả khoảng cách giữa $V_{k}$ và ước lượng cuả nó $V_{k}^{*}$, phản ánh tồn tại cuả QTCN mà đẻ đạt được nó vê mặt kỹ thuật là giới hạn cuà khả năng, hoặc theo quan điểm cuá quan sát viên việc tiếp tục phân biệt là không cần thiết.

Giả sử $-X_{i}, X_{j}$ là hai trạng thái cuả hệ,

thi:

- $\rho: X \times X \rightarrow R, \rho$ là metric cuả hai trạng thái cuả hệ,

- $\epsilon$ - là ngưỡng phân biệt trạng thái cuả hệ.

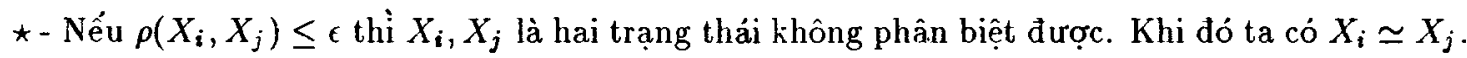

$\star-N e ̂ ́ u \rho\left(X_{i}, X_{j}\right)>\epsilon$ thi $X_{i}, X_{j}$ là hai trạng thái phàn biệt được. Nghĩa là $X_{i} \neq X_{j}$. 
$\rho$ có thể là khỏang cách Euclide:

$$
\rho\left(X_{i}, X_{j}\right)=\left|X_{i}-X_{j}\right|
$$

$\rho$ có thể là độ lệch bình phương. Nếu ta xét không gian các trạng thái là không gian xác suất $(X, \sigma, \mu)$ :

$$
\rho\left(X_{i}, X_{j}\right)=\left[\int_{X_{i}}^{X_{j}}\left(X-X_{i}\right)^{\dot{2}} d P(X)\right]^{1 / 2}
$$

Trong không gian metric điều kiện phân biệt được giữa hai trạng thái $X_{i}, X_{j}$ cuả QTCN tương ứng vơi

$$
\rho\left(X_{i}, X_{j}\right)=\left|X_{i}-X_{j}\right|=\Delta X \geq \epsilon
$$

đồng thời ta có phương sai (dispersion) cuả $X$

$$
D\left(X_{i}\right)=\int_{X}\left(x_{i}-X_{i}\right)^{2} d P\left(x_{i}\right) .
$$

Khi đỏ hiên nhiên điều kiện phân biệt được là [19]

$$
\rho\left(X_{i}, X_{j}\right) \geq \sqrt{D(X)}
$$

Nghĩa là với tư cách là ngưỡng phân biệt có thể lấy trị số

$$
\epsilon=\sqrt{D(X)}
$$

Nếu $X$ là miền biến thiên các trạng thái QTCN thì số lượng lớn nhất các trạng thái có thể phân biệt đối với quan sát viên trong miền đó là

$$
M_{\epsilon}(X)=\frac{|X|}{\epsilon} .
$$

Khi đó theo Ashby, độ da dạng cuà QTCN là

$$
L_{\epsilon}=\log _{2} M_{\epsilon}(X)
$$

Lượng thông tin chứa các đại lượng ngẫu nhiên $x \in X$, xác định trạng thải cuả QTCN hoặc năng ing lı̛ợng cuả nó có trị số lớn nhất chỉ khi các trạng thái (được phân biệt bớ - ngưỡng) là đồng xác ic suất, khi đó

$$
L_{\epsilon}(X)=\max _{p_{i}}\left\{-\sum_{i \in M_{\varepsilon}} p_{i} \log p_{i}\right\}=\max _{p_{i}} H(X)
$$

$p_{i}$ - xác suất cuả trạng thái $x_{i}$, ơ đây $p_{i}=p_{j \neq i} ; \sum_{i \in M_{e}} P_{i}=1: H(X)$ - entropi cuả các đại lượng ngẫu nhiên cuả tỉn hiệu hoặc năng lượng hệ được rời rạc hóa với phân bố $f\left(p_{i}\right)$ và $\epsilon-$ ngưỡng.

Đại lượng $L_{\epsilon}(X)$ ơ (24) gọi là $\epsilon$ - dung lượng thông tin cuả tập $X$ [31]. 
Dịnh lý 3. Giả sứ $\Delta V_{k \cdot m a x}$ là sai số điệu khiển lớn nhất đối với chỉ tiêu chất lượng $k$ cuá QTCN và $\epsilon_{v k}$ là ngưỡng phân biệt đối với $V_{k}$. Nếu $\Delta V_{k \cdot \max } \leq \epsilon_{\eta k}$ thì điều khiển chất lượng QTCN độc lập với điều khiën năng xuất $Q T C N$.

Chứng minh Điều đó có thể dễ dàng rút ra trực tiếp từ khái niệm $\epsilon$ - ngưỡng.

\section{Diều khiến}

Vể bản chát QTCN là một hệ năng lượng [33]. Điểu khiên QTCN là điêu khiên quá trỉnh biến đổi năng lượng (thay đổi mối tương quan giữa động năng và thế năng, biến đổi từ dạng năng lượng này sang dạng năng lượng khác). Ởđây khái niệm năng lượng cần được hiểu rộng hơn. Nó bao gồm sức lao đọng sống, sức lao động đã được vật hóa trong nguyên, nhiên, vật liệu và trong các thiết bị công nghệ cùng với hóa nhiệt năng tích lũy trong vật chất. Khi chọn hàm mục tiêu điểu khiển người ta mong muốn sao cho hiệu quả cuả quá trình đó là lớn nhất (nghia là chọn trạng thải cuả hệ là tối ưu trong những điều kiện ràng buộc nhất định). Khác với các bài toán tối ưu trong quy hoặch toán học, điều khiển QTCN phải được thực hiện trong chế độ thời gian thực và là quả trình đưa hệ từ trạng thái này sang trạng thái khác tốt hơn tiến dần đến trạng thái tối ưu. Khái niệm đó đối với điều khiển QTCN là hoàn toàn hợp lí bởi vì:

a) Trong quản lí xí nghiệp người ta quan tâm đồng thời trạng thái tối uu cuả hệ và trị sổ bằng số đảnh giá hàm mục tiêu (chủ yếu là quan tâm trị số bằng số cuả hàm mục tiêu).

b) Trong điều khiển QTCN vấn đề đặt ra có đội chút khác hơn. Ở đây người ta chị quan tâm đến trạng thái cuả hệ mà ở đó hoặt động cuả hệ là tối ưu. Diêu khiên đó có thể được xác định tì việc giải các bài toán tối ưu mở rộng mà việc giải nó đơn giản hơn việc giải bài toán tối ưu đặt ra ban đầu.

Dịnh nghía 5 Có QTCN được xác định theo các DN 1, 2, 3 và hàm mục tiêu $J(x): \exists x_{j . o p t}(x-$ trạng thái cuả hệ). Bài toán $I(x)$ là bài toán tối uu mở rộng [12] cuả $J(x)$ nếu như

$$
\exists x_{i . o p t}: x_{i . o p t}=x_{j . o p t} .
$$

c) Nếu như không chấp nhận biện pháp nỏi trên thỉ khối lượng lởn thông tin trong điêu khiên QTCN có thể dẫn đén phá vỡ chế độ thời gian thực vì quá tải hệ thống kỹ thuật xử lý thông tin.

Tù những khái niệm trên về trạng thái cuả QTCN rút ra tương ứng các khái niệm điều khiền macro và điều khiền micro:

1- Diểu khiển micro. là tác động lên hệ sao cho các thông số phân tích micro thay đổi theo hướng mong muốn. Bởi vì chất lượng sản phẩm cuối phụ thuộc chủ yếu vào độ chính xác cuả các thông sổ công nghệ, điều khiến micro trước hết thỏa mãn mặt chất lượng sản phẩm. Điều khiên micro do đó có thể thực hiện tự trị đối với tất cà các QTCS cũng như điêu khiển nhiệu liên hệ trong toàn bộ QTCN hoặc đối với tì̀ng nhóm,các QTCS.

2- Diều khiển macro. Trong phân tích macro, aác tính chất macro cuả hệ phụ thuộc vào các tính chất micro và các quan hệ phân cấp. Do đó điều khiên macro là điều khiển cả́c phân bố tính chất micro và các quan hệ phân cấp sao cho sự thay đởi trạng thái cuả hệ (trạng thái macro) xáy ra 
theo hướng tổt hơn. Như vậy, ngoài việc dản nhận chính về mặt sản lượng cuả sản phẩm cuối trong chừng mực nhất định điểu khiển macro có ảnh hưởng đến mặt chất lượng. (Cũng cẩn chú ý rằng giữa điều khiển macro và quản lý xí nghiệp có những khác biệt quan trọng). Trong công nghệ thường hai mặt chất lượng và sản lượng đối lập với nhau. Điểu khiên macro là thực hiện sự thống nhất giữa hai mặt đối lập đó trong sản phẩm cuối. Như vậy, mở rộng $\mathrm{DN} 1$, để thấy rằng điều khiển macro còn là điều kiện tồn tại QTCN.

Từ đó, ta thấy rẳng điểu khiển QTCN là điều khiển đa mục tiêu và được thực hiện ở chể độ dói thoại (dialog) giữa các mức phân cấp cuả hệ. Các thông sổ điểu khiên là các thông số quản lý được, nghĩa là các thông số mà ta có thể thay đởi tiên định (ví dụ các thông số cuà bộ điều chỉnh tự động trong điều khiển micro, các quan hệ có mặt trong đổi thoại giữa các mức phân cấp macro). Trên hỉnh 4 giới thiệu sơ đồ nguyên lý điều khiển QTCN.

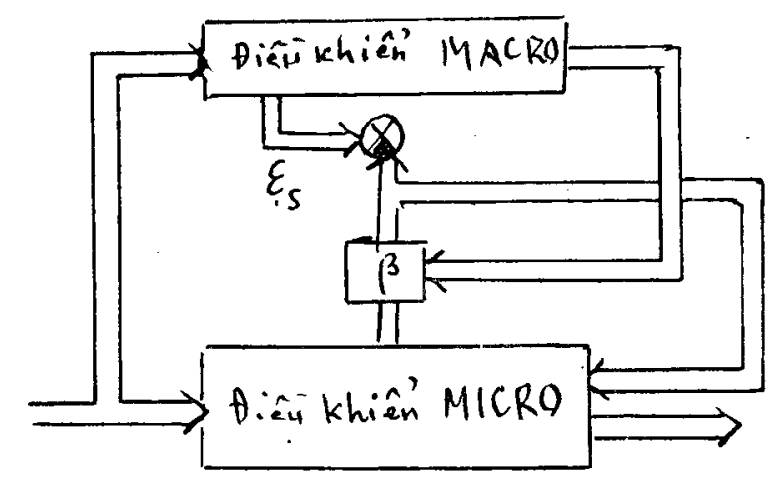

\section{Hình 4 - Sơ đồ lý điều khiển QTCN}

Dịnh nghĩa 6 Cho QTCN xác định theo các ĐN 1,2 và 3 , quá trình điều khiên macro gọi là đối thoại được cẩn thoả mãn điêu kiện (5).

Hệ quà 2 Nếu $\exists \beta_{l}(\neq \emptyset)<<\gamma_{l}$ sao cho $\rho\left(V_{k, \beta_{l}=0}^{*}, V_{k}\right) \leq \epsilon_{k}$ thì có thể bỏ qua đối thoại macro đối với $\mathrm{QTCS}_{l}$. Khi đỏ gọi $\beta_{l}$ là liên hệ yếu.

HQ 2 cho ta them khả năng xây dựng các bài toán tói ưu mớ rộng (26).

\section{Kết luận}

Phương pháp giới thiệu trên đây tỏ ta đơn giản, dễ xử dụng, phù hợp với thực tế công nghệ, cho phép xư dụng có hiệu quả đồng thời các công cụ hiện đại cũng như kinh điển vể lý thuyểt và phương tiện kỹ thuật xứ lý thông tin và điều khiên. Cùng với việc phát triển kỹ thuật vi tỉnh vể khả năng xử lý thông tin (dung lượng nhớ, khả năng thông, tốc độ tỉnh toán) cũng như việc 
giảm đáng kể chi phỉ đầu tư cơ bản cho chúng, tiện cận hệ thống trong điều khiển QTCN ngày càng có ý nghía quan trọng.

\section{Tài liệu tham kháo}

1. Balakirev V.C., Volodin V.M. \& Kirlin A.M., Optimalnoe upravlenie procecami khimicheskoi technology M.1978 ( Tiếng Nga)

2. Baimuratov U.B. \& Kramarenko G.B., Economiseckaia effectivnocts granhixi primenhia visiclitelnoi techniki. Alma-ta .1974. (tiếng Nga )

3. Bakman L.E., Slosnie signali i principi nheopredelennocti v radiolokasi . 1965.(tiếng $\mathrm{Nga}$ ).

4. Duel M.A., Meresko V.M. \& Procketov M.M., AXU teplovoi electrocstansi, Kiev. 1977.(tiếng $\mathrm{Nga}$.

5. Gluskov V.M., Dobrov G.M. \& Teressenko V.I., Besedi ov upravlenhi. 1974. ( tiếng Nga).

6. Zade L.A., Ponhiatie soctoianhia v theorii system . Bài trong sách : "Ovshaia theoria system "M.1966. (tiếng Nga).

7. Kalman P.E., Ov ovsei theorii system upravlenhia, theorii discrestnixoptimalnix i xamonacstraivaiusixsia system. Bài trong sách " Trudi 1 - megdunarodnovo kongrecsa IFAC". M.1963 (tiếng $\mathrm{Nga}$ ).

8. Kuzmin I.V., Asenka effectivnocti i optimizasia ASKU. M.1972.(tiếng Nga).

9. Kuzmin I.V., Iavna A.A. \& Kliusko.V.I., Elementi veroiatnoctnix modelei ASU M 975.(tiếng Nga).

10. Landao L. \& Livsic E.M., Statictiseckaia phisika. M 1964 (tiếng Nga).

11. Lecdon L.C., Optimizasia bolsix system. (Bảng dịch tiếng Nga M 1975.)

12. Chau N.V., Loan N.T. \& Selezuhov M.A. Sintez becspoikovix samonactraivaiusixsia system upravlenhia promislennimi ovektami, ocnovannix na minhimizasii mosnocti signali osivki. Energetika, $\mathrm{n}^{0} 10,1974$ (tiếng Nga).

13. Mamikonov? Upravlenhie $\mathrm{i}$ informasia, M 1975 (tiếng Nga).

14. Mesarovic M. \& Takagiara Ia.- Ovsaia theoria system, matematiseckie ocnovi ( bản dịch tiếng Nga M 1978).

15. Morozovski V.T., Mnogosviaznie systemi avtomatiseckovoe regulirovanhia M1970 (ti)ng Nga).

16. Ocnovi upravlenhia technologiseckimi proseccami, Chủ biên Raiman, N.C. M 1978 (ti)ng Nga).

17. Octrovski G.M. Berezinski T.A.\& Beliaeva A.P. Algoritmi optimizasii chimiko-technologiseckix procecsov. M.1978. (tiếng $\mathrm{Nga}$ ).

18. Padalko L.P., Kriteri i metodi optimalnbvo upravlenhiia electroenergetiseckoi siystemoi, Minsk 1979 (ti)ng Nga).

19. Petrov B.N., Kosubievski I., Ulanov G.M. \& Dudin E.B., Razlisnocst invariantnoc i informasia v systemax s zecskoi i peremennoi structuroi. ( tiếng Nga - bài trong " Trudi III Fsesoiuznoie sovesanhie po avtomatiseckomu upravlenhiu", Technhiseckaia kibernetika, Odesa 1965 \& M 1968).

20. Petrov B.N., Petrov V.V., Ulanov G.M., Ageev V.M., Zaporozes A.V., Kosubievski I.D., Mai 
V.P. \& Uckov A.C., Nachalo informationnoi theorii upravlenhia, .Techniseckaia kibernetika 1968, VNIITI M 1970(tiếng Nga).

21. Petrov E.N., Petrov V.V. \& Ulanov G.M. Nasalo informationnoi theorii upravlenhia ( tiếng Nga, bài trong tuyển tập "Techniseckaia kibernetika " tập 4, chủ biên Petrov B.N. VNIITI M 1972 và trong tạp 6, quyền 1 1975).

22. Problemmi cozdanhia ASU proizvodstvom. Ovzor mezdunarodni centr nausnoi i techniseckoi informasi, M 1977(tiếng Nga).

23. Raibman N.I \& Sadaev V.N., Poctroenhie modelei processov proizvodstva, M 1975(tiếng Nga).

24. Ractrighin L A. Slusainii poics $\mathrm{v}$ processax adaptasi, Riga 1973 (ting Nga).

25. Popirin L.C. Mathematiseckoe modelirovanhie i optimizasia teploenergetiseckix uctanovoc, $M$ 1978 (tiéng Niga).

26. Slosnie systemi upravlenhia, ANUSSR Kiev 1968 (tiếng Nga).

27. Solodov A.Y, Teoria informasii $\mathrm{i}$ co primenhenhie $\mathrm{k}$ nadasam avtomatiseckovo upravlenhia $\mathrm{i}$ kontrolia M 1967 (tiếng $\mathrm{Nga}$ ).

28. Sragovic $V_{a}(3 .$, Teoria adaptivnix system, M 1976 (tiếng Nga).

29. Tikhonov A V. \& Acsenhin V. Ia. Metodi resenhia nlıekorettnix zadast M 1979 (tiếng Nga).

30. Turbovic I.T O sootnoscnhii nheopredelennocti $v$ radiotechnike, Radiotechnika $n^{0} 3,1966$ (tiếng Nga).

31. Ulanov G.M Statictişeckie i informationnie voprocsi upravlenhia po vozmysenhiu, M 1970 (tiếng Nga).

32. Esbi U.P., Vodenhie v kibernetiku. (bản dịch tiếng Nga) M 1959.

33. Chestnut H. - Systems Engineering Tools, John Wiley a Sons Inc, N 1965.

34. Director. S. \& Rohrer R:A., Introduction to Systems Theory N 1972.

35. Handbook of Inductrial Control Computer. Ed. By Harrison T., John wiley a Sons, N. London, Sydney, Toronto 1972.

36. Kullback S., Information Theory and Statistics N.Y. ,London

37. Willson A.G., Entropy in Urban and Regional Modelling Pion Limited London 1970.

38. Simon P. \& Meunier R., Microbiologie industrielle et genie biochimique ( bản dịch tiếng Việt NXB Y học, 1981).

\section{Abstract}

The System Appoach for Technology Process Control

A broad class of technology processes for production of social consumption products in material and energy form is studied by system engineering. The last product is used as existence condition of the mentioned above class. The system of axiom and principal definitions, theorems for structure, analysis and synthesis, observation and control to this class of technology processes is createt. 